I3, 23, on March 3, IO, II, I2, I3, I4, I6, I9, 2I, all on April Io, and on May 3.

In connexion with these phenomena, Mr. Boldermo in Hemnesberget, Ranen, Norway, has made an interesting observation. In a letter to me dated March I2 he says: "About three weeks ago I observed that the northern lights influenced my wireless set. I observed that every time the northern lights appeared against the zenith and on the southern heavens, there was heard a cracking noise in the telephones. ${ }^{2}$ I uncoupled the antenna from the apparatus and the latter appeared to have static charges. Against earth these ran over to a distance of up to $\mathrm{I}_{5} \mathrm{~mm}$. about three times a second. As soon as the northern lights decreased, the charge by degrees disappeared. The night was almost clear with a slight wind. I have observed the same phenomenon twice later on."

In a subsequent letter he gave me a drawing of his antenna, which shows that the top of it is about I 2 metres above the ground. From this it seems that the aurora sometimes modifies enormously the potential gradient in the atmosphere, and this phenomenon should be verified by other observers.

CARL ST\%RMER.

Propagation of a Single Harmonic Wave in a Medium in which the Group Velocity and Wave Velocity are the same.

IT is, I believe, held by many physicists that it is impossible to initiate or transmit a single wave in which the displacement is a simple harmonic function of time and space throughout.

The object of this note is to show that, given certain initial conditions, single waves or finite trains of waves which from beginning to end are of the simple harmonic type can be initiated and will travel unchanged in form; and further that, with slightly modified conditions, fractional parts of such waves can also be propagated unchanged, and will travel with the same velocity as the complete wave of which they would form a part.

Consider first an infinite train of waves in which the displacement referred to the axis $\mathrm{XX}$, Fig. $\mathrm{I}$, is

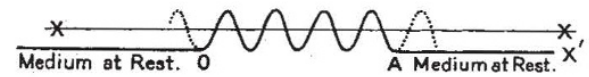

FIG, x.

given by $y=a \sin p a$, where $a$ is the amplitude and $p=2 \pi / \lambda$.

At each maximum and minimum value of $y$ the medium is at rest and the displacement velocity is zero. If the curve is referred to an axis $\mathrm{OX}^{\prime}$, touching the maxima or minima with the origin at $\mathrm{O}$, the equation becomes $y^{\prime}=a\left(x-\cos p x^{\prime}\right)$. At $x^{\prime}=0, \lambda$, $2 \lambda$, etc., the displacement velocity is 0 , and the acceleration $a p^{e}$.

Now suppose $(a)$ that all the waves except those included between $\mathrm{O}$ and $\mathrm{A}$ are removed and that the medium outside these limits is at rest. Then the conditions for the continued propagation of the remaining waves are fulfilled, for the velocities and accelerations within the series remain unchanged, and the terminal accelerations are supplied by the excess of pressure (proportional to $a / \lambda$ ) within the series over that in the medium outside, which is at rest.

(b) If it is required to propagate an odd number of half wave-lengths the medium on either side of the wave series must be at rest, but having pressures

2 That noise was also heard by engineer Max Rich. Hennig in Ångermanland, Sweden, during the aurora on March 9. differing by a quantity proportional to $2 a /$ wavelength. When $(c)$ fractional parts of a wave are to be propagated, not only must the pressures on either side of that part be different, but also the two parts of the medium separated by the wave must have a relative velocity, determined by the displacement velocities at the limits of the propagated fraction.

Case $(a)$, where a single wave or a whole number of waves is under consideration, can easily be made the subject of experiment.

Let a tube of unlimited length and of any diameter (Fig. 2) be provided with a piston on which a harmonic

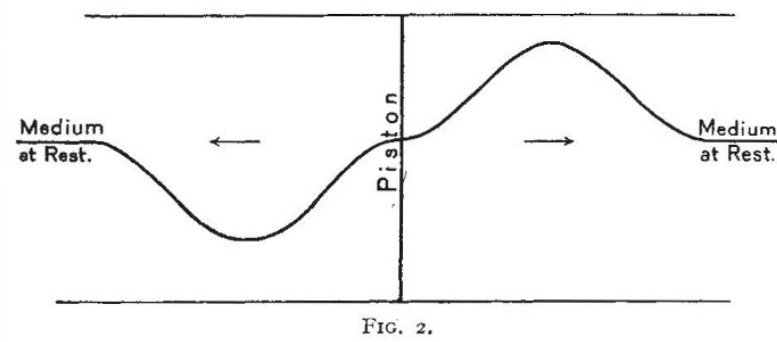

motion can be imposed. Let the air in the tube be at rest and the piston at the position of maximum displacement. If now the piston is caused to make one complete oscillation and to return to rest at its original position, the result will be that a simple harmonic wave will travel along the tube from each side of the piston, and that in one of these waves (namely, that in which the air is at first compressed) the mean pressure will exceed the still air pressure by a quantity proportional to $a / \lambda$, while in the other there will be a defect of pressure of the like amount. A barrier, therefore, placed in the tube will experience an average force during the action of the wave in a direction away from or toward the piston, according as the initiation of the wave starts with an increase or decrease in the air pressure. If the barrier is such as completely to absorb the wave motion, the magnitude of the force will be measured by $a / \lambda$, or by $2 a / \lambda$ if the wave is completely reflected.

In cases $(b)$ and $(c)$, where an odd number of half wave-lengths or fractions of wave-length are concerned, it would be more troublesome to reproduce experimentally the required conditions. In the latter the motion is analogous to, though not identical with, the progress of a 'bore' in a river where the boundary between deep and shallow water travels as a wave with an accompanying change of stream velocity.

9 Baring Crescent,

$$
\text { A. Mallock. }
$$

Exeter.

\section{Barlow's Tables.}

REQUIRING recently the square roots of the numbers from $\mathrm{I}$ to $\mathrm{I}$ oo to ten figures, or one more place of decimals than is given in Barlow, I was led to the discovery that his last figure for numbers above I 250 is not to be depended upon for its accuracy. It is true that De Morgan, in issuing a reprint of Barlow's Tables in 1839 , admitted that his method of checking Barlow's entries by differencing left " remaining a possibility of any of the last figures being a unit wrong in the columns of square and cube roots."

The purpose of this note is not to criticise a work that must ever remain a monument to the industry and zeal of an enthusiastic computer at a time when calculating machines were not in existence, but merely to place on record what must, I am afraid, be a very

NO. 2955, VOL. II 7 ? 